\title{
BMJ Open Values informing the development of an indicator of appropriate diabetes therapy: qualitative study
}

\author{
Christina M LaVecchia,, ${ }^{1,2}$ Victor M Montori, ${ }^{2,3}$ Nilay D Shah, ${ }^{4,5,6}$ \\ Rozalina G McCoy (1) 4,5,7
}

To cite: LaVecchia CM, Montori VM, Shah ND, et al. Values informing the development of an indicator of appropriate diabetes therapy: qualitative study. BMJ Open 2020;10:e044395. doi:10.1136/ bmjopen-2020-044395

- Prepublication history and additional material for this paper are available online. To view these files, please visit the journal online (http://dx.doi. org/10.1136/bmjopen-2020044395).

Preliminary data for this publication were presented as a poster presentation at the American Diabetes Association 79th Scientific Sessions in San Francisco, California, USA (7-11 June 2019).

Received 01 September 2020 Revised 13 November 2020 Accepted 16 November 2020

Check for updates

(C) Author(s) (or their employer(s)) 2020. Re-use permitted under CC BY-NC. No commercial re-use. See rights and permissions. Published by BMJ.

For numbered affiliations see end of article.

Correspondence to Dr Rozalina G McCoy; mccoy.rozalina@mayo.edu

\section{ABSTRACT}

Objectives Despite increasing focus on individualised diabetes management, current diabetes quality measures are based on meeting generic haemoglobin $A_{1 c}$ thresholds and do not reflect considerations of clinical complexity, hypoglycaemic susceptibility or treatment burden. Our team observed a multidisciplinary stakeholder panel tasked with informing an appropriate diabetes therapy indicator (ADTI) and analysed their deliberations, seeking to understand what constitutes appropriate diabetes therapy and how it can be captured using an operational quality indicator. We focused specifically on factors the panel valued in an ideal indicator, how they defined appropriateness and how they thought an indicator of appropriateness could be operationalised.

Design Qualitative study examining Delphi panel deliberations as it iteratively refined the ADTI.

Participants and methods The 12-member panel was comprised of clinicians (endocrinology, primary care, geriatrics), pharmacists, nurses, researchers, and representatives of public and private health plans. It met for four teleconference calls and deliberated asynchronously using semi-structured questionnaires following each call to develop the ADTI. These semistructured questionnaires, as well as the meeting minutes, were then analysed using an inductive thematic approach.

Results We identified three themes in panellist discussions that represented the core value systems underpinning the indicator and its formation: (1) promoting individualised, evidence-based and equitable care; (2) balancing autonomy and prescriptiveness in clinical decision-making; and (3) ensuring an accurate, reliable and practical indicator. These three principles were operationalised into definitions of treatment intensity and clinical complexity, and yielded an indicator that participants judged both fair and effective.

Conclusions Better understanding of what multidisciplinary stakeholders perceive as appropriate diabetes management can help develop quality indicators that are patient-centred, evidence-based, equitable and pragmatic across a range of clinical settings.

\section{INTRODUCTION}

The goal of diabetes management is to prevent immediate (eg, hypoglycaemia and symptomatic hyperglycaemia) and long-term

\section{Strengths and limitations of this study}

- Engagement of a multidisciplinary panel of 12 experts representing diverse backgrounds and perspectives in terms of institutions, professions, gender, and race yielded a rich understanding of the values underpinning appropriate diabetes management and an ideal indicator of diabetes care quality.

- The main limitation of this study is the small number of participants, which may limit the generalisability of study findings.

- We relied on panellists' written responses to a series of open-ended and closed-ended questions disseminated after each call, rather than verbatim recordings of the calls themselves.

- Inferences drawn from this study represent the values of professional experts and may not reflect the values of people living with diabetes.

(eg, microvascular and cardiovascular) complications. In pursuit of this goal, clinical guidelines recommend that glycaemic targets and the medications used to achieve them be individualised to balance the anticipated benefits of these targets with the potential harms of therapy, given each person's clinical and psychosocial situation. ${ }^{1-4}$ At the same time, and in an effort to enable high-quality diabetes care, a variety of performance measures have been introduced for use in public reporting and pay-for-performance reimbursement. Such measures can help identify gaps in care quality, spur and support quality improvement efforts, reward high performers, motivate those lagging behind and inform patients' decisions about their healthcare. Yet, despite the increasing focus on individualisation of diabetes care, ${ }^{1-4}$ current quality measures are based on meeting a generic glycosylated haemoglobin $\left(\mathrm{HbA}_{1 \mathrm{c}}\right)$ threshold $^{56}$ and do not reflect considerations of clinical complexity, hypoglycaemic risk or treatment burden. ${ }^{7}$ In order to develop more patient-centred measures, we sought to understand what would constitute an ideal 
quality measure of appropriate diabetes therapy from the perspective of clinicians, researchers and payers.

Grounding quality measure development in the experience of clinicians directly caring for people with diabetes, experts in diabetes management and payers for healthcare is important for ensuring high-quality care. Yet, quality measures are often developed with minimal input from clinicians and others at the frontlines of care. ${ }^{89}$ This can result in measures that do not reflect the totality and complexity of medical care, ${ }^{8}$ contributing to a potential disconnect between high-quality care and performance on quality measures.

To mitigate these concerns, our team worked to construct an indicator of appropriate diabetes therapy that could more effectively support high-quality and evidence-based care for people with type 2 diabetes across a wide range of clinical complexity. ${ }^{10}$ However, the first step in developing this indicator was to understand how a diverse multidisciplinary team of stakeholders understood the concept of appropriateness and what, to them, constituted a framework for an ideal quality measure of appropriate diabetes therapy. The indicator had to be consistent with the National Academy of Medicine's broad definition of quality as care that is safe, effective, patientcentred, timely, efficient and equitable. ${ }^{11}$ These stakeholders included clinicians, healthcare administrators, researchers and payers. Thus, as the stakeholder panel completed its deliberations on constructing the appropriate diabetes therapy indicator (ADTI), we turned to a qualitative analysis of its process of creation.

Herein, we present this qualitative thematic analysis of the stakeholder panel's deliberations as they discussed and refined this ADTI. We specifically focused on elucidating the factors the panel most valued in an ideal appropriateness indicator, how they defined appropriateness, and how they envisioned an indicator of appropriateness could be operationalised so as to preserve its primary objective of ensuring high-quality, patient-centred and evidence-based care. This analysis also revealed how participants—-stakeholders who may either evaluate with or be evaluated by this indicator-think about quality measures and how the formation process behind the indicator unfolded. Addressing what participants found most important, as well as concerns identified by people with diabetes in subsequent research, may produce a quality indicator that strikes a patient-centred balance between glycaemic control targets and the everyday complexities of being, and treating, people with diabetes.

\section{RESEARCH DESIGN AND METHODS}

This is a qualitative study examining the deliberations of a multidisciplinary team of experts as they came to consensus about what constitutes appropriate glucoselowering therapy for adults with diabetes, how appropriateness can be measured and how such an indicator should be developed. It was conducted and reported in accordance with Standards for Reporting Qualitative Research reporting guidelines. ${ }^{12}$

We worked with OptumLabs to convene a 12-member stakeholder advisory panel to represent diverse perspectives in healthcare, including but not limited to clinicians, data analysts, quality improvement professionals in payer and practice settings, practice or facility administrators, public payers who work with electronic medical record and quality reporting systems, private payers who work with diabetes measure reporting (HEDIS, STAR and so on), patients/consumers or advocates representing the interest of patients with diabetes, employers or other group purchasers. Participants were selected after an open call for nominations (self or other) emailed to all OptumLabs partner institutions (academic and payer) and members of the NQF Measure Incubator Network. Applications were independently reviewed and scored by the principle investigator (RGM) and project manager on the basis of their statement of interest (quality, impact) and expertise (background, knowledge and experience). Twelve of 19 applicants were selected; this number of panellists was a priori defined to be sufficient and practical for informative feedback and productive conversations.

Members provided feedback through a modified Delphi process to help develop and refine the ADTI. ${ }^{10}$ Members (online supplemental table) represented a wide range of areas of expertise and institutional profiles, including clinicians (endocrinology, primary care, geriatrics), pharmacists, nurses, researchers, and representatives of public and private health plans. They also represented diverse gender (four men, eight women) and racial/ethnic (seven White, five non-White) perspectives. Focus group conversations consisted of four 90-minute meetings held over teleconference, facilitated by one of the researchers (RGM) and a project manager, who each kept brief minutes. After each focus group conversation, participants were asked to respond in writing to a range of closed-ended and open-ended questions about the most recent definition of the ADTI and its components; specifically, to reflect on that definition and provide guidance on ADTI definition, framing, and any modifications or changes (please see online supplemental file). Each session covered specific aspects of the indicator, addressed feedback and inquiries from the preceding session, and sought to establish consensus on the indicator definition. Modifications to the traditional Delphi process were as follows: (1) response submissions were not anonymous, though the descriptive data analyses of the panel's responses presented back to the panel were; (2) the panel deliberated on the ADTI definitions during teleconference calls in addition to the asynchronous questionnaires; and (3) the study team provided just-intime information to the panel in response to questions raised in the questionnaires or during teleconference calls in order to assist with ADTI development.

We examined the facilitators' meeting minutes and panellists' written responses using a thematic analysis approach. ${ }^{13-17}$ A qualitative approach allowed us to 
gain insight into the perspectives, attitudes, and values of panel participants, ${ }^{13} 15-17$ who as front-line clinicians, researchers, and payers have had valuable experiences pertinent to quality measurement and implementation. We explored the current and ideal processes of diabetes care quality assessments and what really matters to stakeholders about the quality of diabetes care. The chosen thematic analysis approach provided a flexible, yet rigorous, approach that enabled us to identify salient patterns across our data and draw a range of conclusions $^{15}{ }^{18-20}$ regarding how the indicator development process unfolded, what participants value in high-quality care and what they envision for the final indicator.

Two researchers, a clinician-researcher (RGM) and a qualitative researcher (CML), inductively and independently identified codes, which were refined via consensus and developed in a code book consisting of definitions and example quotations to ensure consistent usage. To yield a trustworthy analysis, both researchers fully coded participants' responses individually. The data were then recoded by both researchers together to establish consensus; any disagreements in coding were resolved verbally. Following multiple rounds of coding the data, the researchers developed themes from the codes. NVivo V.12 (QSR International, Melbourne, Australia) was used for this process to more effectively manage our data and themes and facilitate our understanding of the relationships among our codes, themes and participants. ${ }^{21}$

\section{Patient and public involvement}

This work was motivated by the need for more patientcentred evaluation of diabetes care quality, though not explicitly informed by individual patients' experience and preference. Patients were not directly involved in the design or conduct of this study. Results were communicated to all stakeholder participants on the conclusion of the research and again prior to manuscript submission for publication, and will be broadly disseminated through peer-reviewed publication.

\section{RESULTS}

Working toward an indicator of appropriate diabetes care, the team of panellists focused most intensively on defining what constitutes appropriateness, grounded in scientific evidence and the realities of clinical care and population health management. There was broad consensus that appropriateness reflects care that is neither overly intensive (ie, not overtreatment) nor inadequate (ie, not undertreatment) when considered in the context of each patient's specific situation. Thus, appropriateness necessitates an examination of patient complexity, treatment intensity and treatment outcomes.

The resulting ADTI was both a process indicator (how the patient is treated) and an outcome indicator (what is the final $\mathrm{HbA}_{1 \mathrm{c}}$ level achieved) that was inherently adapted to reflect the patient's underlying complexity. ${ }^{10}$ As the panellists discussed these features of appropriateness, their conversations and written follow-up responses coalesced to reveal three core value systems, which we saw underpinning the development process (as well as the ADTI itself). Those themes were: (1) promoting individualised, evidence-based and equitable care; (2) balancing clinician and patient autonomy with prescriptiveness; and (3) ensuring an accurate, reliable and practical measurement (figure 1). Ultimately, these three principles were operationalised into the panel's definitions of treatment intensity and clinical complexity, and yielded an indicator that participants judged both fair and effective.

\section{Promoting individualised, evidence-based and equitable care}

In discussions invoking this theme, panellists considered ways to ensure the indicator promoted care patterns that are individualised, relevant to each patient with diabetes, consistent with contemporary best practices and scientific evidence, and equitable to all. This theme primarily captured comments made by participants with backgrounds in medicine (three participants), nursing

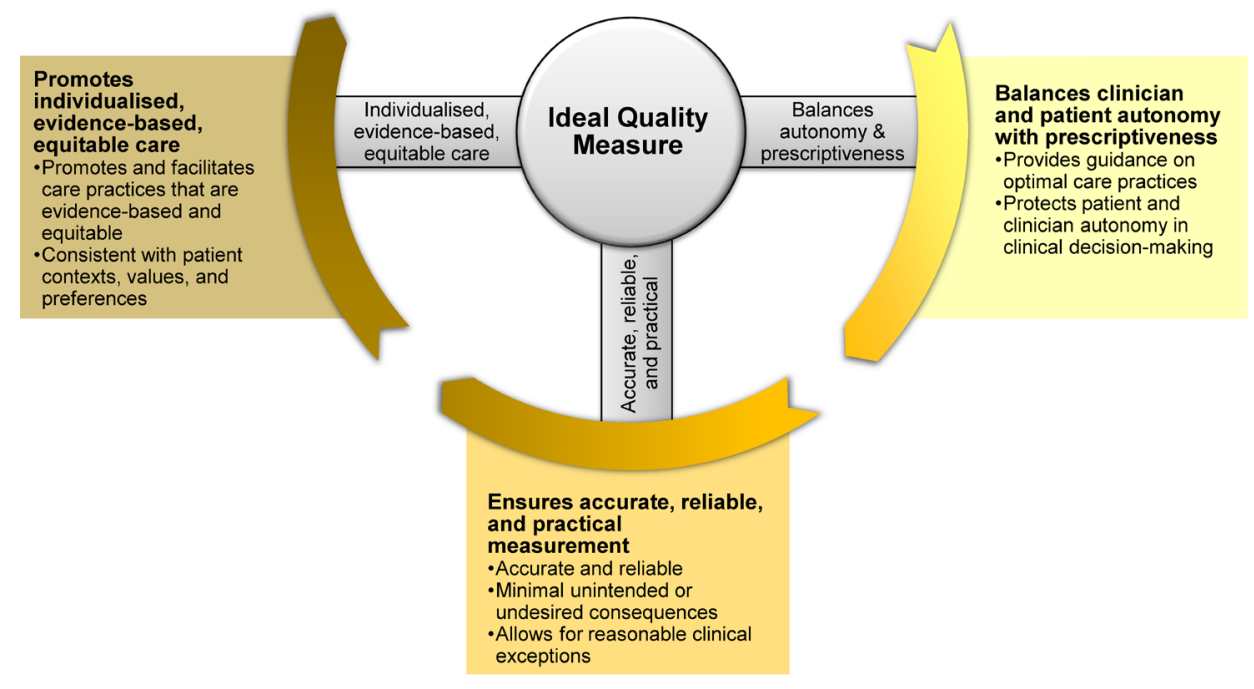

Figure 1 Themes informing an ideal quality indicator. 
Table 1 Participant characteristics and the concerns that came up most frequently in their responses

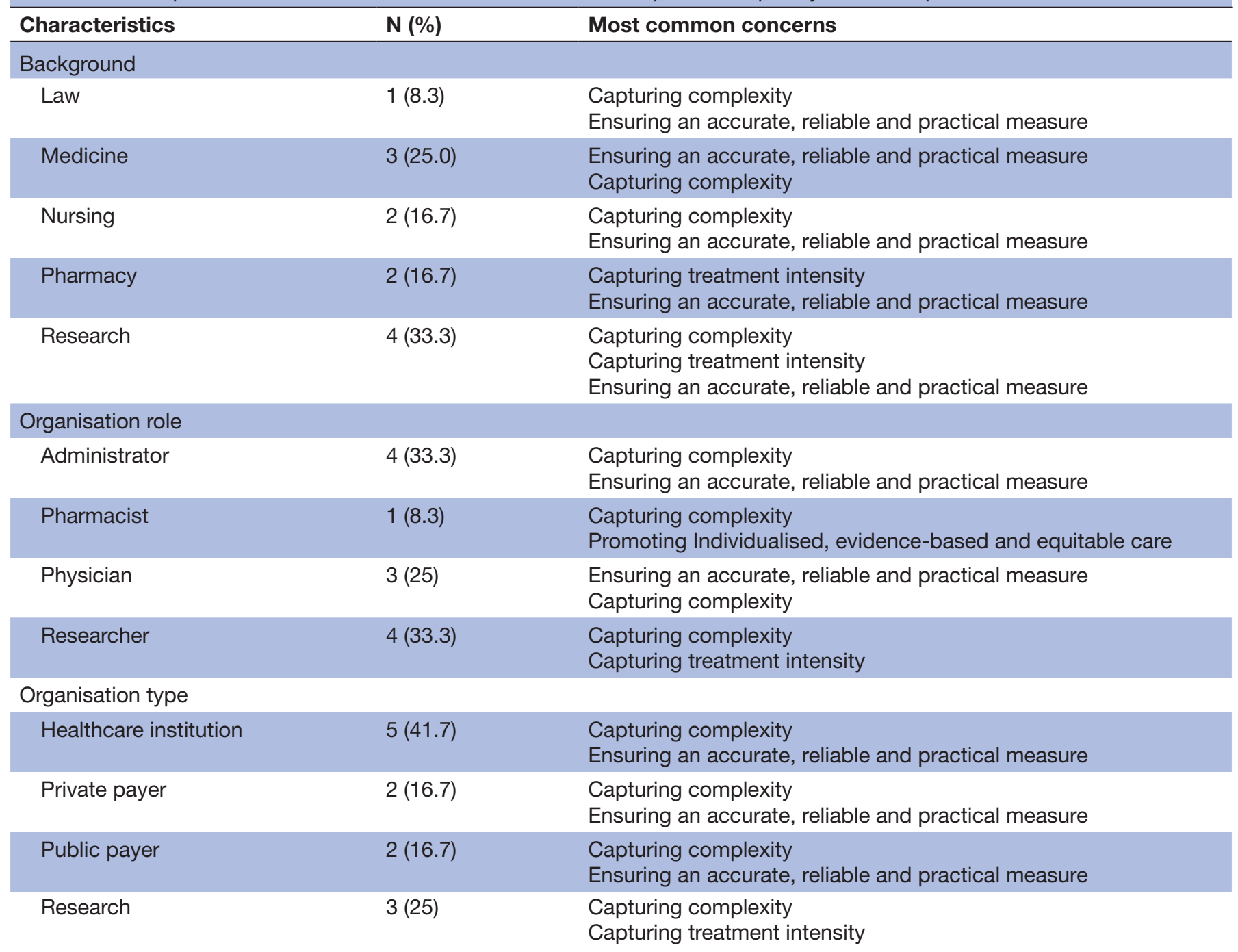

Each of the 12 panel participants was categorised on the basis of three attributes: background, organisation role and organisation type, such that the numerator for each of the three subsections is 12 .

(two participants) and pharmacy (two participants; see table 1).

Panellists focused extensively on ways the ADTI should promote individualised, patient-centred care. As direct quotes provided in table 2 show, participants repeatedly noted that appropriateness should reflect patients' situations, values and preferences for their care. These considerations of patient-centeredness were most attentive to patients' burden of treatment and financial concerns, with some considerations related to comorbidities.

However, while panellists wanted the indicator to address patient preferences, they were mindful that it should not deviate from the best available evidence. They frequently referenced research data, confirming that they wanted the ADTI to maintain consistency with both peer-reviewed literature and consensus opinions (table 2). Similarly, participants demonstrated a desire to reconcile existing clinical practice guidelines with each other and with the indicator. Last, participant responses indicated that they used other quality measures as a guide during the ADTI formation process, using phrases like 'consistent with HEDIS' or 'what we have done with our measures looking at concomitant diagnoses'.

Finally, panellists sought to ensure that the ADTI would be equitable and would not marginalise any groups based on their age, clinical complexity or inability to pay for the preferred treatment choice (table 2).

\section{Balancing clinician and patient autonomy with prescriptiveness}

Panellist responses invoking this theme indicated that they wanted an indicator that would protect the autonomy and agency of both patient and clinician, while simultaneously ensuring that the minimum standard of care is met and reasonable clinical exceptions are allowed. Comments captured by this theme were almost exclusively made by physicians (three participants; table 1). 
Table 2 Selected excerpts from panellists' written responses that informed each theme and the definitions of clinical complexity and treatment intensity

\section{Theme 1: promoting individualised, evidence-based, equitable care}

Individualised $\quad$ "Additional information about the impact of overtreatment, including more specific data about cost and treatment
burden, would be helpful to determine whether reducing overtreatment will have a meaningful impact on patient
outcomes and, relatedly, whether the measure would effective capture provider quality." (emphasis added)
"I am very concerned about the overtreatment definition for patients with low clinical complexity. I don't think the
process of glycemic goal setting is supposed to be isolated to only the physicians' preference."
"While these comorbidities may not be directly related to the clinical effectiveness of glycemic control, they
significantly decrease a patient's ability to be adherent to such a regimen."
"I don't particularly like the term optimal treatment, since without individualized data, it's hard to know what is
optimal."
"CKD stage 4 patients were excluded from ACCORD with the stage 5+ patients."

"Recent CVO trials for GLP-1s and SGLT2s have prompted discussions on combination of dual or triple therapy (metformin plus GLP-1 and/or SGLT2) in patients with established CVD (high clinical complexity patients)."

"The ADA (Standards of Care 2017 page S101) also has a classification called very high complexity which would set an A1c target of $8.5 \%$ as a result of short life expectancy."

Equitable $\quad$ "I have concerns about the use of age as a defining feature of clinical complexity. I think this can lead to
undertreatment in older adults. According to Leal et al.(... Eur Heart J, 2009), the average life expectancy for a type
2 patients who is 75 years is 10 years. So about half of patients with type 2 diabetes at 75 years could benefit from
intensive glycemic control. AGS intentionally does not include age as a criteria for complexity. And the 2017 ADA
Table 11.1 doesn't include age either. I think we should consider removing it."
"In the [American Geriatrics Society] consensus report published in 2012, we did not put age as criteria, as [the]
older population is heterogeneous. Is age important to apply to larger nationwide criteria?(Also, this definition of
clinical complexity has)no mention of functional status. Some older people with multiple comorbidities are highly
functional while others look good on paper but need a lot of caregiving."
"Hypoglycemia should not be the only driver of medication discontinuation-how about a lack of known benefit
with increased costs and other side effects of the medications?"
"If a patient is treated with metformin, sulfonylurea, and NPH at bedtime (because newer medications are too
expensive), wouldn't [they] be considered to be receiving appropriate therapy and not over-treatment?" (emphasis
added).

\section{Theme 2: balancing clinician and patient autonomy with prescriptiveness}

Clinician autonomy "I'm debating with myself whether it would be feasible to allow physicians to exclude patients from this measure someone-like including a code for patients [who] decline changes in medications. I understand that it's possible to change patient's [sic] minds, but I worry about physicians choosing to avoid challenging patients because of this measure."

"In my experience for high clinical complexity patients, some may have long-standing diabetes and significant insulin resistance; they may be on 3 medications to maintain an A1C goal of $<8 \%$ including metformin, sulfonylurea and possibly basal insulin. I think 'dinging' providers here may be inappropriate and management has to be considered on an individual basis."

Patient autonomy "I am very concerned about the overtreatment definition for patients with low clinical complexity. I don't think the process of glycemic goal setting is supposed to be isolated to only the physicians' preference."

\section{Theme 3: ensuring an accurate, reliable and practical measurement}

Accuracy "I think that the logic for $\geq 3$ chronic conditions may be complex for measure [informatic] programming purposes since conditions may potentially be documented with more than one code by different providers. Will there be data sets for the various disease states (ie, one for pulmonary, one for cancer, one for renal disease)? The patient will be required to have one from 3 separate data sets (meaning they can't have three different cancer diagnoses or three different diabetes diagnosis - one has to be from the pulmonary set, one is the cancer set, and one the heart failure set)."

"The proposed measure could be implemented across a variety of settings using data that are collected for other longstanding measures. However, it is not clear whether this measure would take the place of, or complement, endorsed measures related to diabetes control. The impact on provider burden should be explored."

Completeness "I'm a little worried about how these data will be captured. Will it be on the medical center's responsibility to report which patients are excluded based on severe hypo? How could they do that in systems where patients receive care at multiple sites?"

Avoiding unintended consequences

"For example, in a healthy 52 yo who is diagnosed with diabetes and has an A1c of $6.8 \%$, if they are started on metformin and start exercising they could get their $\mathrm{A} 1 \mathrm{C}<5.6 \%$. If their doctor doesn't get the opportunity to stop the metformin, or if the patient prefers to take the metformin (so they don't gain part of the weight back), I wouldn't want to be responsible for penalizing the doctor. I worry that there are similar examples for all of the low complexity overtreatment scenarios." 
Table 2 Continued

Clinical exceptions "I think patients with anemia should be excluded, because the A1Cs are inappropriately low, which could lead to some patients being considered overtreated when they are not being overtreated."

"May consider excluding those who have been hospitalized multiple times during the measurement year. Their A1C measurement becomes irrelevant due to hyperglycemia during illness."

\title{
Coming to consensus: capturing clinical complexity
}

Which comorbidities should be included?

"I think including stage 4 with stage 5 CKD is reasonable, since CKD stage 4 patients can be very prone to hypoglycemia and adverse events."

"The list excludes important other comorbidities which are included in [ADA Standards of Care] Table 11.1 depression, arthritis, falls, incontinence. While these comorbidities may not be directly related to the clinical effectiveness of glycemic control, they significantly decrease a patient's ability to be adherent to such a regimen."

"The ADA (Standards of Care 2017 page S101) also has a classification called very high complexity which would set an A1c target of $8.5 \%$ as a result of short life expectancy ('the presence of a single end-stage chronic illness, such as stage 3-4 congestive heart failure or oxygen-dependent lung disease, chronic kidney disease requiring dialysis, or uncontrolled metastatic cancer, may cause significant symptoms or impairment of functional status and significantly reduce life expectancy')."

"What about including other hospice-level criteria as high complexity, but not as a part of the list of $\geq 3$ chronic conditions. E.g. AIDS, CHF class 4+, metastatic cancer, end-stage liver disease, lung disease on oxygen."

Role of age "I have concerns about the use of age as a defining feature of clinical complexity. I think this can lead to undertreatment in older adults."

"In the(American Geriatrics Society/American Diabetes Association)consensus report published in 2012, we did not put age as criteria, as older population is heterogeneous"

"AGS intentionally does not include age as a criterion for complexity. And the 2017 ADA Table 11.1 doesn't include age either."

"The average life expectancy for a type 2 [diabetes] patient who is 75 years is 10 years. So, about half of patients with type 2 diabetes at 75 years could benefit from intensive glycemic control."

\section{Coming to consensus: capturing treatment intensity}

What treatment approaches are appropriate?

\begin{abstract}
"Recent ADA and AACE discussions on the use of GLP-1 and SGLT-2 as $2^{\text {nd }}$ line for high risk CVD T2D patients. Thus, for CV high risk patients and uncontrolled without use of GLP-1 or SGLT2, it may be defined as 'undertreatment' as the treatment paradigm may be changing over the next few years when the measure is endorsed."

"I think there are cases where basal alone can be low risk and cases where high risk. Same with combination of basal/bolus. My thoughts are that any insulin should be counted as a single medication class. A patient with diabetes managed on basal and bolus insulin wouldn't be considered over-treated just because there are 'two' hypo-prone meds if they are controlled."

"I think the focus should be on hypo-prone vs safer medications instead of number of medications."

"For high clinical complexity, I would think all patients with an A1C $\geq 9 \%$ would be considered to be under-treated because there are still concerns of very high blood sugars even if end of life."
\end{abstract}

AACE, American Association of Clinical Endocrinologists; ADA, American Diabetes Association; AGS, American Geriatrics Society; CHF, congestive heart failure; CKD, chronic kidney disease; CVD, cardiovascular disease; GLP-1, glucagon-like peptide 1; NPH, Neutral Protamine Hagedorn; SGLT2, sodium-glucose transport protein 2.

Responses reveal that the panel was concerned about how the ADTI would impact clinicians' abilities to individualise treatment and exercise clinical decision-making and/or actions (table 2). For example, when prompted to choose language for treatment modalities in the indicator, one participant stated a preference for terms that 'give clinicians some room for individualisation'. Another noted concerns about the indicator's impact on lower complexity patients. When asked to elaborate on a scenario she used to express her concerns, she reflected: "I was thinking about how to give space for clinical judgement without adding ambiguity. It's something I have struggled with and don't have an answer [for]."

In parallel, panellists demonstrated concern as to whether the ADTI would impact the ability of patients to have a say in their care (table 2). Meeting minutes additionally documented a concern that $\mathrm{HbA}_{1 \mathrm{c}}$ targets fail to capture patient preferences.

\section{Ensuring an accurate, reliable and practical measurement}

This final theme shows participants were attentive to ensuring ease of implementing the quality indicator in clinical practice, safeguarding accuracy and reliability, and avoiding unintended effects or punitive functions. Comments under this theme, which typically examined whether the indicator was fair and reliable, were the most numerous in our data. Physicians (three participants) by far contributed most to discussions that fell under this theme, followed by those with backgrounds in research (four participants) and nursing (two participants) (table 1). 
Many of the comments about the practicalities of implementation considered elements like time, effort and burden (table 2). For example, participants discussed how comorbidities and medications would be defined and captured across different clinical settings. Other comments were concerned with documentation, both in terms of reasonable accuracy ("I think the 12-months period preceding the last A1c will be a better guide as health status change frequently in older population") and completeness (making sure that all relevant comorbidities and treatment modifiers would be captured). Finally, participants reiterated why it is important to allow for clinical exceptions in ADTI application and enforcement, and considered the potential implications of introducing this quality indicator into practice. They often invoked this theme through scenarios illustrating the kinds of unintended effects for patients and clinicians they wanted to avoid (table 2).

In parallel, participants expressed a desire to reduce unexpected effects the ADTI may have on treatment decisions and patient outcomes, particularly if these might lead to clinically suboptimal care. They also wanted to preclude any malfeasance by clinicians and/or organisations, whereby performance on the indicator would outweigh the quality of care delivered.

\section{Coming to consensus on appropriate and individualised/ patient-centered diabetes management}

These three themes-promoting individualised, evidence-based and equitable care; balancing autonomy and prescriptiveness; and ensuring an accurate, reliable and practical indicator-were used to inform the definition of appropriate and patient-centred diabetes care. The primary task of the expert panel was to come to consensus on what constitutes appropriate, overly intensive, and inadequate glucose-lowering therapy for people with diabetes across a wide range of clinical, personal and societal contexts. Because no such classification existed to date, all conversations centred on the precise definitions of two important concepts: clinical complexity (ie, in what situations would patients warrant less intensive glucose-lowering therapy?) and treatment intensity (ie, what treatment regimens would be considered overly intensive or inappropriately relaxed across the newly defined clinical complexity levels?).

\section{Capturing clinical complexity}

When defining clinical complexity, panellists discussed a range of issues, including the types of comorbidities that contribute to complexity, how to define multimorbidity or clinical complexity, and how age may factor into the definition of clinical complexity (table 2). While participants wanted to relax treatment goals and targets for older adults, as recommended by clinical guidelines, ${ }^{22-24}$ they were also wary of potentially undertreating otherwise healthy older patients. They ultimately made their final recommendations based on empirical data. ${ }^{25}$
Overwhelmingly, participants brought up the third theme (ensuring an accurate, reliable and practical indicator) when discussing complexity, reflecting their concerns about the technical aspects of implementing the proposed definitions in an efficient, yet still accurate, way. For example, they noted that 'dementia can be poorly documented' and 'the logic for $\geq$ three chronic conditions may be complex for measure programming purposes'.

\section{Capturing treatment intensity}

For treatment intensity, participants discussed whether medication use should be allowed or even promoted independent of glucose-lowering properties, including among clinically complex patients who may not benefit from their glucose-lowering effects (table 2). This related specifically to promoting, not penalising, the use of glucagon-like peptide-1 (GLP-1) receptor agonists and sodium-glucose transport protein 2 (SGLT2) inhibitors by patients with cardiovascular or kidney disease even at lower $\mathrm{HbA}_{1 \mathrm{c}}$ levels. Participants also discussed whether there may be situations where all patients are considered as undertreated, no matter how clinically complex they are or how intensively they are treated. Citing clinical guidelines, the panel endorsed considering patients with $\mathrm{HbA} 1 \mathrm{c} \geq 9.0 \%$ as potentially undertreated and benefiting from interventions to address gaps in diabetes care quality.

Participants most frequently invoked the first theme (promoting individualised, evidence-based and equitable care) when discussing treatment intensity, specifically with regard to patient situations. In other words, discussions around treatment intensity commonly referenced patients' circumstances, goals, and preferences for treatment as a rationale for including different treatment intensities and/or targets in the indicator. Finally, they sought to develop an indicator that would be durable to emerging scientific evidence and best practices, stating that "it would be good for us to think about the meaning and relevance of the measure in the near future."

\section{DISCUSSION}

To better understand the motivations, reasoning and aspirations underpinning what healthcare professionals perceive to be a patient-centred quality indicator of appropriate diabetes therapy, we thematically analysed the written feedback of the multidisciplinary expert panel that was tasked with developing it. We focused specifically on identifying what the panel valued, prioritised and operationalised in such an indicator. Our themes revealed several points of significance, the foremost being the nuance within which medicine operates and the challenge of ascribing a firm value statement (eg, appropriateness) to a dynamic construct at the confluence of patient preference, medical and psychosocial patient context, evolving scientific literature and realities of everyday practice. Ultimately, the three core value 
systems defining an ideal quality indicator that emerged from stakeholder deliberations were: (1) promoting individualised, evidence-based and equitable care; (2) balancing autonomy and prescriptiveness in clinical decision-making; and (3) ensuring an accurate, reliable and practical measurement.

The panel sought to develop an indicator that would reflect, facilitate, and reward treatment practices that are, in essence, high quality: safe, effective, patient-centred, timely, efficient and equitable. ${ }^{11}$ This balanced approach is primarily reflected by the first theme of panellist deliberations ('promoting individualised, evidence-based and equitable care'). Importantly, individualisation was construed to weigh each patient's clinical complexity (number and severity of comorbidities, symptom burden, burden of treatment, life expectancy), psychosocial complexity and capacity to adhere to a potentially complex treatment regimen. Panellists recognised that treatment costs and financial barriers to care should be considered, though were cautious not to have a separate standard of care for lower income or under/uninsured patients.

The first theme further reveals that panellists were conflicted about the role chronological age should play in defining clinical complexity. While they recognised the correlation between advanced age and life expectancy, panellists-particularly those with clinical training-were clear that older adults are a heterogeneous population and the balance of benefit/harm from an intensive therapeutic approach should be evaluated on a case-by-case basis. Finally, panellists spoke at length about equity and fairness in the ADTI definition and its implementation, ensuring that patients and their clinicians are not disadvantaged on the basis of their age, race, gender or clinical status. We were surprised, however, that most discussions about fairness related to patient age and financial means, rather than implicit or systemic bias that may exist toward women and minority patients.

The second theme ('balancing autonomy and prescriptiveness in clinical decision-making') shows the desire to honour the centrality of the patient-clinician relationship. That relationship is predicated on preserving the autonomy and agency of both patients and clinicians so that they can come to a shared understanding about the most appropriate care. We see this as an attempt to operationalise shared decision-making, with mutual concern for both patients and clinicians, rather than a paternalistic regard for clinicians or an overcorrective focus on patients that burdens rather than empowers. ${ }^{26-28}$ The ADTI therefore seeks to encourage patients and clinicians to make decisions that best fit patients' lives practically, intellectually and emotionally. And yet, woven into the discussions about shared decision-making were references to clinical guidelines and randomised controlled trials, calling for some temperance of patient and clinician autonomy by the data and expert opinion. This also represented a strong desire by the panellists to conform to existing and evolving guidelines formulated by experts who were not in the 'room'.
Responses from this theme also reinforced some concerns raised in the literature regarding the quality measurement process. ${ }^{29}$ They were made primarily by physician panellists, who drew on their personal experiences in practice to inform the indicator development and evaluation process. Most concerns involved situations that may penalise clinicians for delivering evidence-based care that a quality indicator may deem to be 'inappropriate' (eg, a patient with heart failure prescribed an SGLT2 inhibitor despite having a low/normal $\mathrm{HbA}_{1 \mathrm{c}}$ or a patient who cannot tolerate metformin and needs to be treated with a sulfonylurea due to inability to afford other nonhypoglycaemia-prone medications) or where appropriateness cannot be assessed (eg, patients with anaemia or recent hospitalisation for whom $\mathrm{HbA}_{1 \mathrm{c}}$ measurement may not be accurate). Our analysis showed that participants tried to account for clinical and non-clinical exceptions to the ADTI, although within the constraints of a practical quality indicator that would be informative and not burdensome on the practice. This balance of the clinical ideal juxtaposed against the realities of modern healthcare permeated throughout the panel's deliberations.

The third theme ('ensuring an accurate, reliable and practical measurement') centred on the practical and logistical aspects of operationalising the ADTI in the realworld setting. Panellists focused on developing concise definitions that could be implemented across a wide range of practices without undue burden, ensuring accuracy and reliability of the indicator, and minimising unintended consequences. This theme included the practical 'how' of quality measurement, including which data are to be captured, how they are to be tracked seamlessly and in real-time, and how they can be best reported. Participants, particularly physicians, were concerned about how the indicator may penalise clinicians not meeting it due to factors outside their control. We were surprised to find little resistance to the need for measurement and reporting more broadly, though clinicians in particular sought to protect the ADTI from being misapplied for better perceived performance at the expense of patient health and well-being. They also felt it to be best used as a quality indicator and a mechanism for quality improvement, not for public reporting and performance-based reimbursement.

These themes informed the operational definition of the ADTI and considerations of appropriateness ${ }^{10}$ which weighed who the patient is (capturing complexity), what is done to the patient (capturing treatment intensity), and what can be reliably and reasonably documented by clinicians and extracted by the health system. Panellists further sought to ensure that the indicator would be informative to and actionable by clinicians, providing practical suggestions for actions that clinicians can take to improve care and health outcomes. The final indicator therefore included not only its definition, but also a menu of suggested management items for patients who are flagged as potentially undertreated, overtreated or appropriately treated. In our analysis, the discussions of 
clinical complexity were more nuanced and frequent than discussions of treatment intensity, which is appropriate as patient complexity can often be considered by clinicians as something not under their control.

Moreover, participants recognised the many nonmedical factors in patients' lives outside the clinical domain that can create complexity. Discussions about treatment intensity similarly recognised nonpharmacological approaches to diabetes management and the importance of addressing social determinants of health. Panellists stopped short of recommending that non-pharmacological approaches be included as a 'treatment' in the indicator definition, largely due to practical considerations about what can be reasonably documented in the electronic health record and be within the clinician's purview to implement, particularly for patients with financial and logistical barriers to lifestyle therapies (eg, healthy food, safe space for exercise). Still, this presents a missed opportunity to reward non-pharmacological approaches to care, including social support (eg, referrals to social services, community health workers, food banks), lifestyle management (eg, dietetics and exercise programmes) and mental health. Nevertheless, panellists stressed that the ADTI implicitly promoted non-pharmacological therapy, particularly for the clinically complex, because it would enable better glycaemic control with fewer medications and hence would promote 'appropriate' care. Future development of other measures should similarly include discussions of non-pharmacological interventions when discussing appropriate care.

The main limitation of this study is the relatively small number of participants on the panel, though for its initial goal of forming the ADTI itself, the team deemed the panel to be a sufficient and practical size for informative feedback and productive conversations. Further, the 12 selected panellists represented diverse backgrounds and perspectives in terms of institutions, professions, gender and race. While our sample size may limit the generalisability of study findings to all quality measures, this illustrative case reveals some of the core values that informed our quality indicator and thus lends insight into the formation of quality measures more broadly. Second, because our primary objective in convening the panel was to develop a patient-centred indicator of appropriate diabetes therapy (ie, the ADTI), and it was not until the rich conversations with the panel occurred that we discovered how helpful and productive it may be for others to learn from these deliberations, we did not capture the content of each conference call verbatim. Instead, we relied on panellists' written responses to a series of open-ended and closedended questions disseminated after each call, as these represented the most direct and comprehensive feedback each individual participant provided on the indicator based on their experience and expertise. The panellists' thoughtful and extensive feedback on ADTI development and the implications of its potential implementation into clinical practice therefore provide a window into their individual perspectives. Finally, while patient perspectives and feedback were considered when developing the indicator, contents of a patient focus group were not included in this analysis.

Themes elicited from the deliberations of this multidisciplinary expert panel reinforce the importance of individualised care, scientific evidence, the patient-clinician relationship and practicality. In other words, an ideal indicator-and good care-lie at the intersection of patients, scientific evidence and reality. While any type of measure could meet these criteria, we envision a hybrid indicator that is comprised of both process (to reward what the clinician is doing) and outcome (to reward preferred health outcomes) metrics that allow for partial, rather than all-or-none, success and has both upper and lower bounds of acceptability as clinically appropriate. As the healthcare system shifts to performance-based reimbursement, population health, and alterative care delivery models that prioritise quality and value (eg, Accountable Care Organizations, Patient-Centered Medical Homes), quality measurement will be increasingly important. The themes elicited herein provide a framework for characteristics that healthcare professionals, payers and quality indicator development experts view as important in an ideal patient-centred quality indicator.

\section{Author affiliations}

${ }^{1}$ School of Arts and Sciences, Neumann University, Aston, Pennsylvania, USA ${ }^{2}$ Knowledge and Evaluation Research (KER) Unit, Mayo Clinic, Rochester, Minnesota, USA

${ }^{3}$ Division of Endocrinology, Diabetes, Metabolism, and Nutrition, Department of Medicine, Mayo Clinic, Rochester, Minnesota, USA

${ }^{4}$ Division of Health Care Policy \& Research, Department of Health Sciences Research, Mayo Clinic, Rochester, Minnesota, USA

${ }^{5}$ Mayo Clinic Robert D. and Patricia E. Kern Center for the Science of Health Care Delivery, Rochester, MN, United States

${ }^{6}$ OptumLabs, Cambridge, MA, United States

${ }^{7}$ Division of Community Internal Medicine, Department of Medicine, Mayo Clinic, Rochester, Minnesota, USA

Contributors RGM is the guarantor of this work and, as such, had full access to all the data in the study and takes responsibility for the integrity of the data and the accuracy of the data analysis. RGM and CML designed the study, interpreted the data and co-wrote the manuscript. VMM and NDS contributed to the discussion and reviewed/edited the manuscript.

Funding This effort was funded by an AARP Quality Measure Innovation Grant through a collaboration with OptumLabs and the NQF Measure Incubator (RGM) and the National Institute of Health National Institute of Diabetes and Digestive and Kidney Diseases grant number K23DK114497 (RGM).

Disclaimer Study contents are the sole responsibility of the authors and do not necessarily represent the official views of $\mathrm{NIH}$.

\section{Competing interests None declared.}

Patient consent for publication Not required.

Ethics approval Mayo Clinic Institutional Research Board deemed this study exempt from review, as participants were subject matter experts who provided general recommendations for quality indicator development and the project posed minimal risk of harm to participants.

Provenance and peer review Not commissioned; externally peer reviewed.

Data availability statement Anonymised documents used for qualitative analyses are included in the Supplement. Codebook used for the analyses is available upon reasonable request by emailing RGM (mccoy.rozalina@mayo. edu). 
Supplemental material This content has been supplied by the author(s). It has not been vetted by BMJ Publishing Group Limited (BMJ) and may not have been peer-reviewed. Any opinions or recommendations discussed are solely those of the author(s) and are not endorsed by BMJ. BMJ disclaims all liability and responsibility arising from any reliance placed on the content. Where the content includes any translated material, BMJ does not warrant the accuracy and reliability of the translations (including but not limited to local regulations, clinical guidelines, terminology, drug names and drug dosages), and is not responsible for any error and/or omissions arising from translation and adaptation or otherwise.

Open access This is an open access article distributed in accordance with the Creative Commons Attribution Non Commercial (CC BY-NC 4.0) license, which permits others to distribute, remix, adapt, build upon this work non-commercially, and license their derivative works on different terms, provided the original work is properly cited, appropriate credit is given, any changes made indicated, and the use is non-commercial. See: http://creativecommons.org/licenses/by-nc/4.0/.

\section{ORCID iD}

Rozalina G McCoy http://orcid.org/0000-0002-2289-3183

\section{REFERENCES}

1 NICE. National Institute for health and care excellence pathways: managing blood glucose in Aults with type 2 diabetes: National Institute for health and care excellence, 2019. Available: https:// pathways.nice.org.uk/pathways/type-2-diabetes-in-adults [Accessed 23 Apr 2019].

2 Garber AJ, Abrahamson MJ, Barzilay Jl, et al. Consensus statement by the american association of clinical endocrinologists and american college of endocrinology on the comprehensive type 2 diabetes management algorithm - 2019 executive summary. Endocr Pract 2019;25:69-100.

3 Conlin PR, Colburn J, Aron D, et al. Synopsis of the 2017 U.S. department of Veterans Affairs/U.S. Department of defense clinical practice guideline: management of type 2 diabetes mellitus. Ann Intern Med 2017;167:655-63.

4 American Diabetes Association. 6. Glycemic Targets: standards of medical care in diabetes-2020. Diabetes Care 2020;43:S66-76.

5 MNCM. Minnesota community measurement data collection guide: optimal diabetes care specifications, 2019 report year (01/01/2018 to 12/31/2018 dates of service). Minneapolis, MN; 2018.

6 NCQA. Comprehensive Diabetes Care: Hemoglobin A1c (HbA1c) Control (<8.0\%). National Quality Forum (NQF)-endorsed measure \#0575. Measure steward: National Committee for Quality Assurance. Available: http://www.qualityforum.org/QPS/MeasureDetails.aspx? standardID=944\&print=0\&entityTypelD=1 [Accessed 21 Feb 2020].

7 Rodriguez-Gutierrez R, McCoy RG. Measuring what matters in diabetes. JAMA 2019;321:1865

8 Aloia TA, Jackson T, Ghaferi A, et al. Developing minimally invasive procedure quality metrics: one step at a time. Surg Endosc 2019;33:679-83.

9 Baernholdt M, Dunton N, Hughes RG, et al. Quality measures: a Stakeholder analysis. J Nurs Care Qual 2018;33:149-56.
10 McCoy RG, Lipska KJ, Van Houten HK, et al. Development and evaluation of a patient-centered quality indicator for the appropriateness of type 2 diabetes management. BMJ Open Diabetes Res Care 2020;8:e001878.

11 IOM. Institute of medicine, Committee on quality of health care in America: crossing the quality chasm: a new health system for the 21st century. Washington, DC; 2001.

12 O'Brien BC, Harris IB, Beckman TJ, et al. Standards for reporting qualitative research: a synthesis of recommendations. Acad Med 2014;89:1245-51.

13 Strauss AL. Qualitative analysis for social scientists. Cambridge Cambridgeshire ; New York: Cambridge University Press, 1987.

14 ed.Saldaña J. The coding manual for qualitative researchers. third edition. Los Angeles, London: SAGE, 2016.

15 Braun V, Clarke V. Using thematic analysis in psychology. Qual Res Psychol 2006;3:77-101.

16 Pope C, van Royen P, Baker R. Qualitative methods in research on healthcare quality. Qual Saf Health Care 2002;11:148-52.

17 Fitzpatrick R, Boulton M. Qualitative methods for assessing health care. Qual Health Care 1994:3:107-13.

18 Braun V, Clarke V. What can "thematic analysis" offer health and wellbeing researchers? Int J Qual Stud Health Well-being 2014;9:26152-52.

19 Castleberry A, Nolen A. Thematic analysis of qualitative research data: is it as easy as it sounds? Curr Pharm Teach Learn 2018;10:807-15.

20 Chafe $\mathrm{R}$. The value of qualitative description in health services and policy research. Healthc Policy 2017;12:12-18.

21 Jackson K, Bazeley P. Qualitative data analysis with NVivo. Third Edition. SAGE Publications Ltd, 2019.

22 American Diabetes Association. 12. Older Adults: Standards of Medical Care in Diabetes-2020. Diabetes Care 2020;43:S152-62

23 Kirkman MS, Briscoe VJ, Clark N, et al. Diabetes in older adults: a consensus report. J Am Geriatr Soc 2012;60:2342-56.

24 LeRoith D, Biessels GJ, Braithwaite SS, et al. Treatment of diabetes in older adults: an endocrine Society* clinical practice guideline. $J$ Clin Endocrinol Metab 2019;104:1520-74.

25 McCoy RG, Lipska KJ, Van Houten HK, et al. Association of cumulative multimorbidity, glycemic control, and medication use with Hypoglycemia-Related emergency department visits and hospitalizations among adults with diabetes. JAMA Netw Open 2020;3:e1919099-e99.

26 Kunneman M, Montori VM, Castaneda-Guarderas A, et al. What is shared decision making? (and what it is not). Acad Emerg Med 2016;23:1320-4.

27 Nickel WK, Weinberger SE, Guze PA, et al. Principles for patient and family partnership in care: an American College of physicians position paper. Ann Intern Med 2018;169:796-9.

28 Politi MC, Dizon DS, Frosch DL, et al. Importance of Clarifying patients' desired role in shared decision making to match their level of engagement with their preferences. BMJ 2013;347:f7066.

29 Kerr EA, Krein SL, Vijan S, et al. Avoiding pitfalls in chronic disease quality measurement: a case for the next generation of technical quality measures. Am J Manag Care 2001;7:1033-43. 